\title{
Asymptotic Rational Approximation To Pi: Solution of an "Unsolved Problem" Posed By Herbert Wilf
}

\author{
Mark Daniel Ward $\rrbracket^{\dagger}$ \\ Department of Statistics, Purdue University, 150 North University St., West Lafayette, IN 47907-2067, USA
}

\begin{abstract}
The webpage of Herbert Wilf describes eight Unsolved Problems. Here, we completely resolve the third of these eight problems. The task seems innocent: find the first term of the asymptotic behavior of the coefficients of an ordinary generating function, whose coefficients naturally yield rational approximations to $\pi$. Upon closer examination, however, the analysis is fraught with difficulties. For instance, the function is the composition of three functions, but the innermost function has a non-zero constant term, so many standard techniques for analyzing function compositions will completely fail. Additionally, the signs of the coefficients are neither all positive, nor alternating in a regular manner. The generating function involves both a square root and an arctangent. The complex-valued square root and arctangent functions each rely on complex logarithms, which are multivalued and fundamentally depend on branch cuts. These multiple values and branch cuts make the function extremely tedious to visualize using Maple.

We provide a complete asymptotic analysis of the coefficients of Wilf's generating function. The asymptotic expansion is naturally additive (not multiplicative); each term of the expansion contains oscillations, which we precisely characterize. The proofs rely on complex analysis, in particular, singularity analysis (which, in turn, rely on a Hankel contour and transfer theorems).
\end{abstract}

Keywords: analytic combinatorics, asymptotic analysis, rational approximation, generating function, singularity analysis

\section{Introduction}

The webpage of Wilf (2009) contains a pdf file of eight "Unsolved Problems". These include questions about, respectively: series for $\pi$; growth of partition functions; a problem in asymptotics; the quadratic character of binomial coefficients; Young tableaux; distinct multiplicities (among parts of partitions); Toeplitz determinants; and chromatic number. These problems are extremely interesting to read and to think about. We completely resolve the third of these eight problems:

\footnotetext{
${ }^{\dagger}$ M. D. Ward’s research was supported by NSF Science \& Technology Center grant CCF-0939370. 


A problem in asymptotics Wilf (2009)
Let
\[ f(z)=\frac{\arctan \sqrt{2 e^{-z}-1}}{\sqrt{2 e^{-z}-1}} . \]
If $f(z)=\sum_{n \geq 0} a_{n} z^{n}$, find the first term of the asymptotic behavior of the $a_{n}$ 's.

One reason that the behavior of the coefficients of $f(z)$ is so interesting is that each coefficient can be written in the form $a_{n}=b_{n} \pi-c_{n}$ where $b_{n}$ 's and $c_{n}$ 's are nonnegative rational numbers. For example:

\begin{tabular}{|c|c|}
\hline$n$ & $a_{n}$ \\
\hline \hline 0 & $\frac{1}{4} \pi$ \\
1 & $\frac{1}{4} \pi-\frac{1}{2}$ \\
2 & $\frac{1}{4} \pi-\frac{3}{4}$ \\
3 & $\frac{7}{24} \pi-\frac{11}{12}$ \\
4 & $\frac{35}{96} \pi-\frac{55}{48}$ \\
5 & $\frac{113}{240} \pi-\frac{71}{48}$ \\
6 & $\frac{1787}{22880} \pi-\frac{2807}{1440}$ \\
7 & $\frac{16717}{20160} \pi-\frac{8753}{3630}$ \\
8 & $\frac{2257}{2016} \pi-\frac{94541}{26880}$ \\
9 & $\frac{315883}{207360} \pi-\frac{694663}{145152}$ \\
10 & $\frac{4324721}{2073600} \pi-\frac{47552791}{7257600}$ \\
\hline
\end{tabular}

In fact $\lim _{n \rightarrow \infty} a_{n} / b_{n}=0$ and thus the rational numbers of the form $c_{n} / b_{n}$ provide approximations to $\pi$.

Wilf's 3rd Unsolved Problem is to find the precise first term asymptotic behavior of the $a_{n}$ 's. The analysis of $f(z)$ is surprisingly very challenging, for several reasons:

1. The function $f(z)$ is the composition of three simpler functions, $\frac{\arctan z}{z}, \sqrt{z}$, and $2 e^{-z}-1$. Unfortunately, the innermost function, $2 e^{-z}-1$, has a non-zero constant term. In general, when the innermost function in a composition has a non-zero constant term, many of the standard analytic combinatorics techniques completely fail. See, for instance, Section VI.9, "Functional composition," in Flajolet and Sedgewick (2009).

2. The coefficients $a_{n}$ are not all positive. The signs of the $a_{n}$ 's alternate between positive and negative in an erratic, non-systematic way. This prevents the analysis of $f(z)$ using many techniques in enumerative combinatorics that require the coefficients to be all positive or to systematically alternate between positives and negatives.

3. The definition of $f(z)$ involves a square root and an arctangent. Each of these functions are defined over the complex numbers using complex logarithms, which are multivalued and fundamentally depend on branch cuts. These multiple values and choices of branch cuts make the function extremely tedious to visualize using the Maple 14 symbolic algebra software.

The method of analysis in this paper follows the precept of Painlevé (1900) and Hadamard (1945): questions posed about real numbers can often be resolved using the methods of complex analysis. Flajolet and Sedgewick (2009) and Szpankowski (2001) give systematic and comprehensive treatments about the use of analytic (complex) techniques for many types of asymptotic analysis of algorithms, data structures, 
and other combinatorial and probabilistic applications. We rely on the techniques of singularity analysis, as discussed in Flajolet and Sedgewick (2009).

\section{Main Results}

Let $g(z)=\sqrt{2 e^{-z}-1}$. The definition of the square root function in the complex plane is ambiguous, up to a possible change of sign, so we clarify the definition of $g(z)$ very precisely. Let

$$
\mathcal{S}_{j}=\{x+\mathrm{i} y \mid x, y \in \mathbb{R} \text { with } 2 j \pi<y<2(j+1) \pi\} \cup\{x+2 j \pi \mathrm{i} \mid x \in \mathbb{R} \text { with } x<\ln (2)\} .
$$

The regions $\mathcal{S}_{j}$ are disjoint and have the following property:

$$
\bigcup_{j \in \mathbb{Z}} \mathcal{S}_{j}=\mathbb{C} \backslash \bigcup_{j \in \mathbb{Z}} \mathcal{L}_{j}
$$

where $\mathcal{L}_{j}$ is defined as

$$
\mathcal{L}_{j}=\{z=x+2 j \pi \mathrm{i} \mid x \in \mathbb{R}, \ln (2) \leq x\} .
$$

(In Lemma 2.1. we will show that $f(z)$ has an analytic extension to $\bigcup_{j \in \mathbb{Z}} \mathcal{S}_{j}$.) Now we define $g(z)$ very precisely:

$$
g(z)= \begin{cases}-\exp \left(\frac{1}{2} \log \left(2 e^{-z}-1\right)\right) & \text { for } z \in \mathcal{S}_{j}, \text { where } j \text { is an even integer } \\ +\exp \left(\frac{1}{2} \log \left(2 e^{-z}-1\right)\right) & \text { for } z \in \mathcal{S}_{j}, \text { where } j \text { is an odd integer }\end{cases}
$$

here, we define $\log \left(2 e^{-z}-1\right)$ unambiguously by the following branch of logarithm: Write $2 e^{-z}-1=$ $r e^{\mathrm{i} \theta}$ for $r \in \mathbb{R}^{>0}$ and $0 \leq \theta<2 \pi$, and then unambiguously define $\log \left(2 e^{-z}-1\right)=\ln r+\mathrm{i} \theta$.

For $\arctan z=\frac{\mathrm{i}}{2} \log \frac{\mathrm{i}+z}{\mathrm{i}-z}$, write $\frac{\mathrm{i}+z}{\mathrm{i}-z}=r e^{\mathrm{i} \theta}$ for $r \in \mathbb{R}^{>0}$ and $-2 \pi<\theta \leq 0$, and then define $\log \frac{\mathrm{i}+z}{\mathrm{i}-z}=\ln r+\mathrm{i} \theta$. This choice of the branch of arctan in the numerator of $f(z)$ allows for an analytic extension to $\mathcal{L}_{0}$ (as well as, automatically, an analytic extension to $\mathcal{L}_{j}$ for all even $j$ ). In contrast, with this choice of branch of arctan, $f(z)$ cannot be analytically extended to $\mathcal{L}_{j}$ for odd $j$. So $R=|\ln (2)+2 \pi \mathrm{i}|$ is the maximum radius around the origin for which $f(z)$ has an analytic extension, because the closest singularities to the origin will be at $\zeta=\ln (2)+2 \pi \mathrm{i}$ and its complex conjugate $\bar{\zeta}=\ln (2)-2 \pi \mathrm{i}$. The asymptotic analysis of $f(z)$ using analytic methods relies on having the largest possible disc, centered at the origin, on which $f(z)$ is analytic. So this choice of the branch of arctan is crucial for the analysis that follows later, in Theorem 2.4 .

Lemma 2.1 The function $g(z)=\sqrt{2 e^{-z}-1}$ is analytic on $\mathbb{C} \backslash \bigcup_{j \in \mathbb{Z}} \mathcal{L}_{j}$.

Lemma 2.2 The function $f(z)$ is analytic on $\mathbb{C} \backslash \bigcup_{j \in \mathbb{Z}} \mathcal{L}_{j}$. Moreover, an appropriate choice for the branch of the arctan function allows $f(z)$ to have an analytic continuation to $\bigcup_{j \in 2 \mathbb{Z}} \mathcal{L}_{j}$; however, such an analytic continuation will leave $f(z)$ discontinuous on $\bigcup_{j \in 2 \mathbb{Z}+1} \mathcal{L}_{j}$.

Figure 1 illustrates the region on which $f(z)$ is analytic, as in Lemma 2.2

Theorem 2.3 Let $a_{n}$ denote the coefficient of $z^{n}$ in the generating function $f(z)=\frac{\arctan \sqrt{2 e^{-z}-1}}{\sqrt{2 e^{-z}-1}}$, namely, $f(z)=\sum_{n \geq 0} a_{n} z^{n}$. Then

$$
a_{n}=R^{-n} h(n),
$$

where $R=|\ln (2)+2 \pi \mathrm{i}|$, and $h(n)$ is a subexponential factor, i.e., $\lim _{\sup } \rightarrow \infty|h(n)|^{1 / n}=1$. 


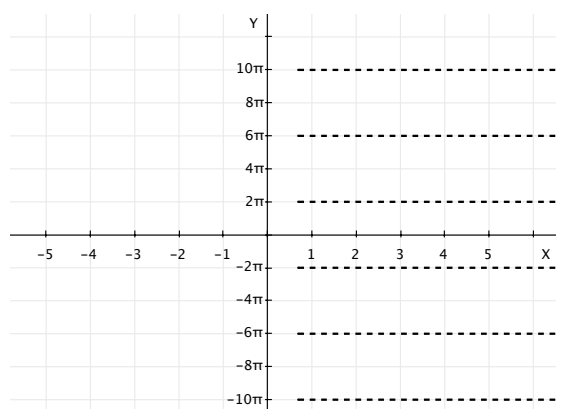

Fig. 1: Region of the complex plane for which $f(x+\mathrm{i} y)$ is analytic. The closest singularities to the origin are located at $\zeta=\ln (2)+2 \pi \mathrm{i}$ and its complex conjugate $\bar{\zeta}=\ln (2)-2 \pi \mathrm{i}$. So the radius of convergence is $R=|\zeta|$, and $\mathcal{C}_{R}:=\{z|| z \mid<R\}$ is the largest open disc around the origin on which $f(z)$ is analytic.

Rigorous proofs of Lemma 2.1. Lemma 2.2 and Theorem 2.3 are given in Section 3 . The veracity of Theorem 2.3 is also evident in Figure2. Using $a_{n}$ as in Theorem 2.3, the Maple plot in Figure2 2 reinforces our understanding that $\limsup _{n \rightarrow \infty}|h(n)|^{1 / n}=1$. To fully complete the solution of Wilf's problem, we

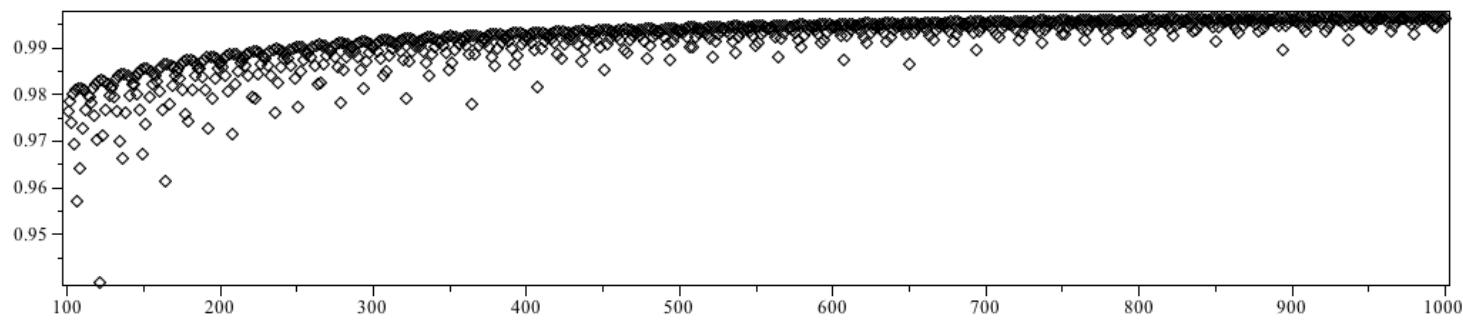

Fig. 2: Maple plot of $|h(n)|^{1 / n}=R\left|a_{n}\right|^{1 / n}=|\ln (2)+2 \pi \mathrm{i}|\left|\left[z^{n}\right] f(z)\right|^{1 / n}$ for $100 \leq n \leq 1000$.

must precisely describe the first term of the asymptotic behavior of $h(n)$ too. As we see in Theorem 2.4 . $a_{n}$ is oscillating; this is also apparent in the figures.

Theorem 2.4 gives the complete description of the asymptotic behavior of the $a_{n}$ 's. We follow the notation from pages 381-384 of Flajolet and Sedgewick (2009) in the definitions of $e_{k}$ and $\lambda_{k, \ell}$.

Theorem 2.4 Let $a_{n}$ denote the coefficient of $z^{n}$ in the generating function $f(z)=\frac{\arctan \sqrt{2 e^{-z}-1}}{\sqrt{2 e^{-z}-1}}$, namely, $f(z)=\sum_{n \geq 0} a_{n} z^{n}$. Then for each positive integer $N$,

$a_{n}=-2 \pi \sum_{j=0}^{N-1} d_{j} \frac{\cos ((n-j+1 / 2) \arctan (2 \pi / \ln (2)))}{\Gamma(-j+1 / 2) R^{n-j+1 / 2} n^{j+1 / 2}}\left(1+\sum_{k=1}^{N-j-1} \frac{e_{k}\left(\frac{1}{2}-j\right)}{n^{k}}\right)+O\left(\frac{1}{R^{n} n^{N+1 / 2}}\right)$,

where $d_{j}=\left[z^{j-1 / 2}\right]\left(e^{z}-1\right)^{-1 / 2}$, and $e_{k}(\alpha)=\sum_{\ell=k}^{2 k}(-1)^{\ell} \lambda_{k, \ell}(\alpha-1)(\alpha-2) \cdots(\alpha-\ell)$, and $\lambda_{k, \ell}=$ $\left[v^{k} t^{\ell}\right] e^{t}(1+v t)^{-1-1 / v}$, and $R=|\ln (2)+2 \pi \mathrm{i}|$. 
For instance, when $N=3$ in Theorem 2.4.

$$
\begin{aligned}
a_{n}=-2 \sqrt{\pi} & {\left[\frac{\cos ((n+1 / 2) \arctan (2 \pi / \ln (2)))}{R^{n+1 / 2}}\left(\frac{1}{\sqrt{n}}-\frac{1}{8 n^{3 / 2}}+\frac{1}{128 n^{5 / 2}}\right)\right.} \\
& +\frac{\cos ((n-1 / 2) \arctan (2 \pi / \ln (2)))}{R^{n-1 / 2}}\left(\frac{1}{8 n^{3 / 2}}+\frac{3}{64 n^{5 / 2}}\right) \\
& \left.+\frac{\cos ((n-3 / 2) \arctan (2 \pi / \ln (2)))}{R^{n-3 / 2}} \frac{3}{384 n^{5 / 2}}\right] \\
& +O\left(\frac{1}{R^{n} n^{7 / 2}}\right) .
\end{aligned}
$$

We prove Theorem 2.4 in Section 3 In addition to a formal proof, we include several figures that give credence to Theorem 2.4

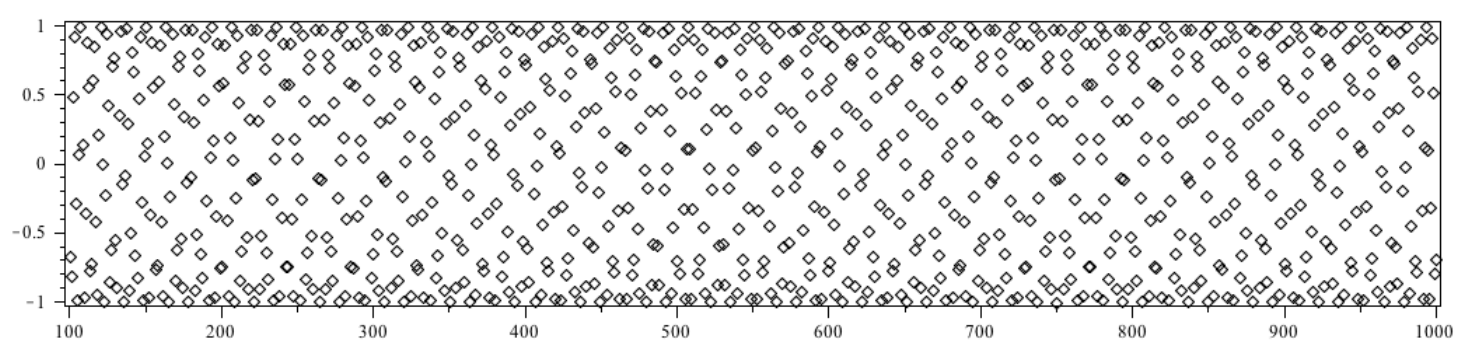

Fig. 3: Maple plot of $-\frac{\sqrt{n} R^{n+1 / 2} a_{n}}{2 \sqrt{\pi}}$ for $100 \leq n \leq 1000$.

Figure 3 depicts the fact that the first additive term of the asymptotic behavior of $a_{n}$ is $-\frac{2 \sqrt{\pi}}{\sqrt{n} R^{n+1 / 2}}$ multiplied by some oscillating term, i.e., Theorem 2.4 gives the correct additive first order of $a_{n}$. For other examples of such oscillations in asymptotic terms, see for instance: the section "Nonperiodic fluctuations" (pages 264-266) or note VI.4 (pages 384-385) both from Flajolet and Sedgewick (2009). Figure 4 exhibits the normalized difference between $a_{n}$ and the first three terms of the asymptotic expansion, from Theorem 2.4. Figures 5 and 6 illustrate the fact that the asymptotic expansion of $a_{n}$ must be expressed additively, not multiplicatively. In other words, it is not true that $\frac{a_{n}}{-2 \sqrt{\pi} \frac{\cos ((n+1 / 2) \arctan (2 \pi / \ln (2)))}{\sqrt{n} R^{n+1 / 2}}} \rightarrow 1$. Since the cosine is occasionally extremely close to 0 (as seen by the spikes in Figures 5 and 6, the lower order terms of the asymptotic expression will occasionally dominate the (otherwise) higher order terms. These figures help provide insight into the asymptotic properties of $a_{n}$.

\section{Proofs}

Proof: (of Lemma 2.1 For $z$ in the interior of $\mathcal{S}_{j}$, the function $g(z)=\sqrt{2 e^{-z}-1}$ is the composition of analytic functions and is thus also analytic. So we only need to check that $f(z)$ is continuous and analytic on the boundary between $\mathcal{S}_{j}$ and $\mathcal{S}_{j-1}$, i.e., for $z=x+2 j \pi$ i with $x \in \mathbb{R}^{<\ln (2)}$. We claim

$$
\lim _{\substack{t \rightarrow z \\ t \in \mathcal{S}_{j-1}}} g(t)=g(z) \quad \text { and } \quad \lim _{\substack{t \rightarrow z \\ t \in \mathcal{S}_{j-1}}} g^{\prime}(t)=g^{\prime}(z) \text {. }
$$




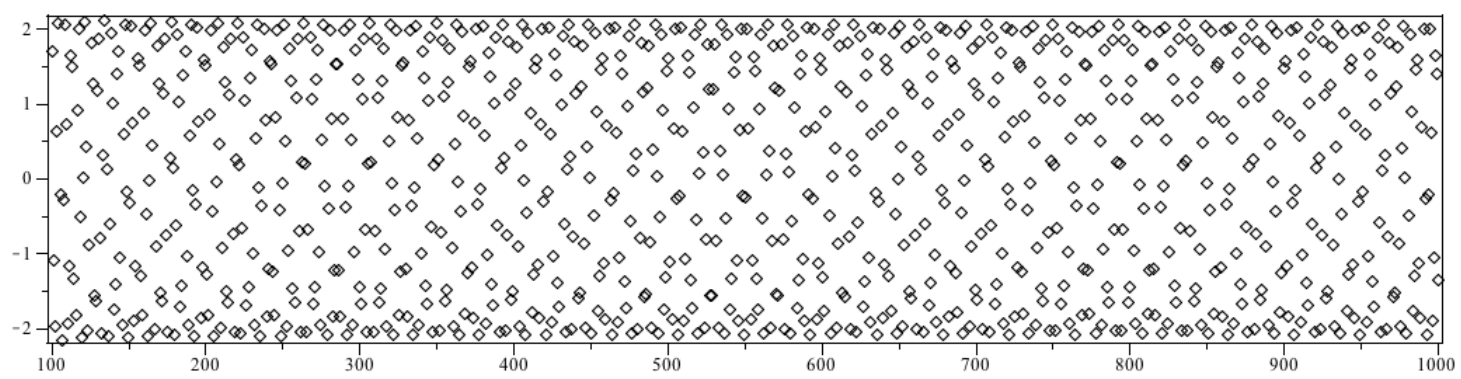

Fig. 4: Maple plot of $R^{n} n^{7 / 2}\left(-2 \sqrt{\pi}\left[\frac{\cos ((n+1 / 2) \arctan (2 \pi / \ln (2)))}{R^{n+1 / 2}}\left(\frac{1}{\sqrt{n}}-\frac{1}{8 n^{3 / 2}}+\frac{1}{128 n^{5 / 2}}\right)+\right.\right.$ $\left.\left.\frac{\cos ((n-1 / 2) \arctan (2 \pi / \ln (2)))}{R^{n-1 / 2}}\left(\frac{1}{8 n^{3 / 2}}+\frac{3}{64 n^{5 / 2}}\right)+\frac{\cos ((n-3 / 2) \arctan (2 \pi / \ln (2)))}{R^{n-3 / 2}} \frac{3}{384 n^{5 / 2}}\right]-a_{n}\right)$ for $100 \leq n \leq 1000$.

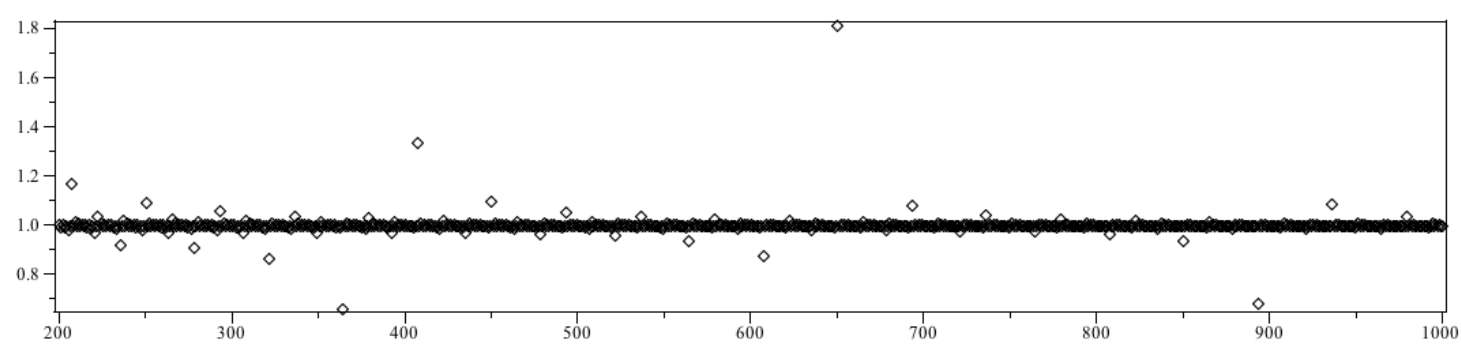

Fig. 5: Maple plot of $\frac{a_{n}}{-2 \sqrt{\pi} \frac{\cos ((n+1 / 2) \arctan (2 \pi / \ln (2)))}{\sqrt{n} R^{n+1 / 2}}}$ for $200 \leq n \leq 1000$.

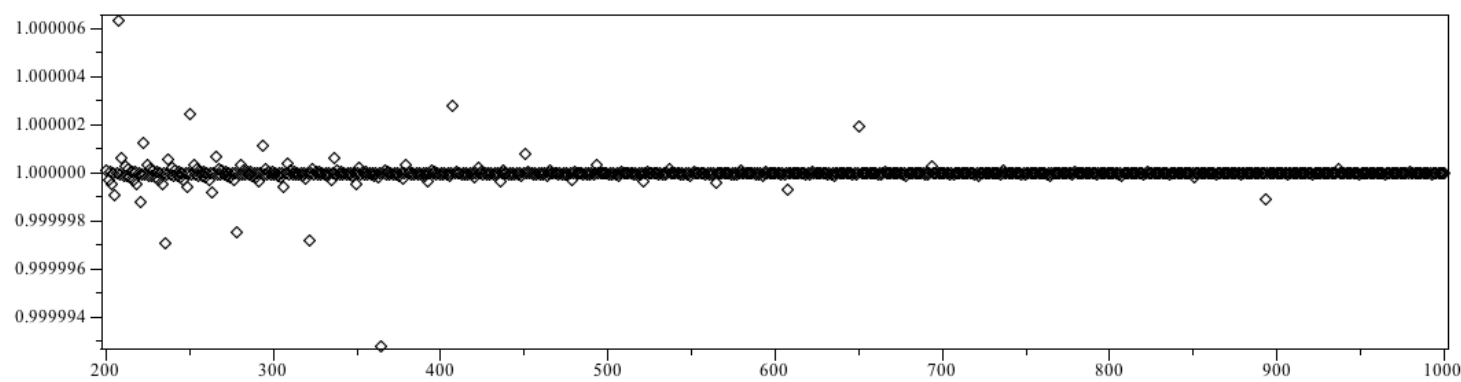

Fig. 6: Maple plot of $a_{n}$ divided by $-2 \sqrt{\pi}\left[\frac{\cos ((n+1 / 2) \arctan (2 \pi / \ln (2)))}{R^{n+1 / 2}}\left(\frac{1}{\sqrt{n}}-\frac{1}{8 n^{3 / 2}}+\frac{1}{128 n^{5 / 2}}\right)+\right.$ $\left.\frac{\cos ((n-1 / 2) \arctan (2 \pi / \ln (2)))}{R^{n-1 / 2}}\left(\frac{1}{8 n^{3 / 2}}+\frac{3}{64 n^{5 / 2}}\right)+\frac{\cos ((n-3 / 2) \arctan (2 \pi / \ln (2)))}{R^{n-3 / 2}} \frac{3}{384 n^{5 / 2}}\right]$ for $200 \leq n \leq 1000$. 
(These properties are already clear for $t \in \mathcal{S}_{j}$, so we only check for $t \in \mathcal{S}_{j-1}$.) Define $r, r_{t} \in \mathbb{R}^{>0}$ and $\theta \in \mathbb{R}^{\geq 0}$ such that $2 e^{-t}-1=r_{t} e^{\mathrm{i} \theta_{t}}$ and $2 e^{-z}-1=r \in \mathbb{R}^{>0}$. Then $r_{t} \rightarrow r$ and $\theta_{t} \rightarrow 0$, so $\log \left(2 e^{-t}-1\right)=\ln \left(r_{t}\right)+\mathrm{i} \theta_{t} \rightarrow \ln (r)+0$ i. Thus $\exp \left(\frac{1}{2} \log \left(2 e^{-t}-1\right)\right) \rightarrow \exp \left(\frac{1}{2} \ln (r)\right)=\sqrt{r}$. So

$$
\begin{aligned}
& g(t)=\sqrt{2 e^{-t}-1}=\exp \left(\frac{1}{2} \log \left(2 e^{-t}-1\right)\right) \rightarrow \sqrt{r}=g(z), \quad \text { for } j \text { even; } \\
& g(t)=\sqrt{2 e^{-t}-1}=-\exp \left(\frac{1}{2} \log \left(2 e^{-t}-1\right)\right) \rightarrow-\sqrt{r}=g(z), \quad \text { for } j \text { odd. }
\end{aligned}
$$

Thus $g(z)$ is continuous at $z$. Since $g^{\prime}(z)=\left(-\frac{1}{2}+e^{-z} g(z)\right) /\left(2 e^{-z}-1\right)$, then $g^{\prime}(t) \rightarrow g^{\prime}(z)$. Thus, $g(z)$ is indeed analytic on $\mathbb{C} \backslash \bigcup_{j \in \mathbb{Z}} \mathcal{L}_{j}$.

Proof: (of Lemma 2.2 The set $\{z=\mathrm{i} y \mid y \in \mathbb{R},-1 \leq y \leq 1\}$ is sent by the conformal map $z \mapsto \frac{\mathrm{i}+z}{\mathrm{i}-z}$ to the line $\left\{z=x \mid x \in \mathbb{R}^{\geq 0}\right\}$; otherwise the image of $z \mapsto \frac{\mathrm{i}+z}{\mathrm{i}-z}$ is disjoint from $\left\{z=x \mid x \in \mathbb{R}^{\geq 0}\right\}$. Therefore, $\log \frac{\mathrm{i}+z}{\mathrm{i}-z}$ is analytic on the set $z \notin\{z=\mathrm{i} y \mid y \in \mathbb{R},-1 \leq y \leq 1\}$ (i.e., the branch cut is avoided). Since $g(z)$ is analytic for $z \in \mathbb{C} \backslash \bigcup_{j \in \mathbb{Z}} \mathcal{L}_{j}$ (shown in Lemma 2.1, and the range of $g(z)$ is disjoint from $\{z=\mathrm{i} y \mid y \in \mathbb{R},-1 \leq y \leq 1\}$, it follows that $f(z)=\frac{\arctan g(z)}{g(z)}=\frac{\frac{\mathrm{i}}{2} \log \frac{\mathrm{i}+g(z)}{\mathrm{i}-g(z)}}{g(z)}$ is the composition of analytic functions and is thus also analytic for $z \in \mathbb{C} \backslash \bigcup_{j \in \mathbb{Z}} \mathcal{L}_{j}$.

Let $j$ be an even integer. We prove that $f(z)$ has an analytic continuation to $\mathcal{L}_{j}$. Each $z \in \mathcal{L}_{j}$ can be written in the form $z=x+2 j \pi$ i for $x \in \mathbb{R}^{\geq \ln (2)}$. Then $2 e^{-z}-1 \in \mathbb{R}^{\leq 0}$. We define

$$
f(z)=\frac{\frac{1}{2} \ln \frac{1+\sqrt{-\left(2 e^{-z}-1\right)}}{1-\sqrt{-\left(2 e^{-z}-1\right)}}}{\sqrt{-\left(2 e^{-z}-1\right)}} \text { for } z=x+2 j \pi \mathrm{i} \text { where } x \in \mathbb{R}^{>\ln (2)} ; \text { and also } f(\ln (2)+2 j \pi \mathrm{i})=1 \text {. }
$$

Consider $z \in \mathcal{S}_{j}$ with $z \neq \ln (2)+2 j \pi$ i. If $t \in \mathcal{S}_{j}$ and with $t \rightarrow z$, then $g(t) \rightarrow-\mathrm{i} \sqrt{-\left(2 e^{-z}-1\right)}$ so

$$
f(t)=\frac{\frac{\mathrm{i}}{2} \log \frac{\mathrm{i}+g(t)}{\mathrm{i}-g(t)}}{g(t)} \rightarrow \frac{\frac{1}{2} \ln \frac{1-\sqrt{-\left(2 e^{-z}-1\right)}}{1+\sqrt{-\left(2 e^{-z}-1\right)}}}{-\sqrt{-\left(2 e^{-z}-1\right)}}=\frac{\frac{1}{2} \ln \frac{1+\sqrt{-\left(2 e^{-z}-1\right)}}{1-\sqrt{-\left(2 e^{-z}-1\right)}}}{\sqrt{-\left(2 e^{-z}-1\right)}}
$$

Similarly, for $t \in \mathcal{S}_{j-1}$ with $t \rightarrow z$, we have $g(t) \rightarrow i \sqrt{-\left(2 e^{-z}-1\right)}$ so

$$
f(t)=\frac{\frac{\mathrm{i}}{2} \log \frac{\mathrm{i}+g(t)}{\mathrm{i}-g(t)}}{g(t)} \rightarrow \frac{\frac{1}{2} \ln \frac{1+\sqrt{-\left(2 e^{-z}-1\right)}}{1-\sqrt{-\left(2 e^{-z}-1\right)}}}{\sqrt{-\left(2 e^{-z}-1\right)}} .
$$

Also, $f(z)$ is continuous at $z=\ln (2)+2 j \pi \mathrm{i}$, and the derivative of $f(z)$ exists and is continuous (all of this is straightforward to check), so the definition in (1) is an analytic continuation of $f(z)$ for $z \in \mathcal{L}_{j}$.

For odd $j$, an additional analytic continuation of $f(z)$ to $\mathcal{L}_{j}$ is not possible. Consider $z=x+2 j \pi \mathrm{i}$ for $x \in \mathbb{R}$ with $x>\ln (2)$. Then $2 e^{-z}-1 \in \mathbb{R}^{<0}$. Thus, for $t \in \mathcal{S}_{j}$ with $t \rightarrow z$, we have $g(t) \rightarrow$ $i \sqrt{-\left(2 e^{-z}-1\right)}$ so

$$
f(t)=\frac{\frac{\mathrm{i}}{2} \log \frac{\mathrm{i}+g(t)}{\mathrm{i}-g(t)}}{g(t)} \rightarrow \frac{\frac{1}{2}\left(\ln \frac{1+\sqrt{-\left(2 e^{-z}-1\right)}}{1-\sqrt{-\left(2 e^{-z}-1\right)}}-2 \pi\right)}{\sqrt{-\left(2 e^{-z}-1\right)}}=\frac{\frac{1}{2} \ln \frac{1+\sqrt{-\left(2 e^{-z}-1\right)}}{1-\sqrt{-\left(2 e^{-z}-1\right)}}-\pi}{\sqrt{-\left(2 e^{-z}-1\right)}} .
$$


Similarly, for $t \in \mathcal{S}_{j-1}$ with $t \rightarrow z$, we have $g(t) \rightarrow-\mathrm{i} \sqrt{-\left(2 e^{-z}-1\right)}$ so

$$
f(t)=\frac{\frac{\mathrm{i}}{2} \log \frac{\mathrm{i}+g(t)}{\mathrm{i}-g(t)}}{g(t)} \rightarrow \frac{\frac{1}{2}\left(\ln \frac{1-\sqrt{-\left(2 e^{-z}-1\right)}}{1+\sqrt{-\left(2 e^{-z}-1\right)}}-2 \pi\right)}{-\sqrt{-\left(2 e^{-z}-1\right)}}=\frac{\frac{1}{2} \ln \frac{1+\sqrt{-\left(2 e^{-z}-1\right)}}{1-\sqrt{-\left(2 e^{-z}-1\right)}}+\pi}{\sqrt{-\left(2 e^{-z}-1\right)}} .
$$

Thus, $f(z)$ is not continuous, and thus not analytic, at $z=x+2 j \pi \mathrm{i}$ for odd integers $j$ and $x>\ln (2)$. Similarly, $f(z)$ is discontinuous at $\ln (2)+2 j \pi$ i for odd $j$.

Proof: (of Theorem 2.3 By Lemma 2.2 the radius of convergence of $f(z)$ about the origin is exactly $R=$ $|\zeta|=|\ln (2)+2 \pi \mathrm{i}|$. Indeed, on the boundary of $\mathcal{C}_{R}$, the function $f(z)$ has non-removable singularities exactly at $z=\zeta=\ln (2)+2 \pi \mathrm{i}$ and $z=\bar{\zeta}=\ln (2)-2 \pi \mathrm{i}$. So the radius of convergence of $f(z)$ about the origin is exactly $R$. The classical Exponential Growth Formula (see, e.g., Flajolet and Sedgewick (2009) provides a natural correspondence between the radius of converge of any function $f(z)$ and the rate of growth of the function's coefficients: If $f(z)$ is analytic at all points in an open disc of radius $r$ about the origin, but has a non-removable singularity on the boundary, then the coefficients $a_{n}=\left[z^{n}\right] f(z)$ satisfy $a_{n}=r^{-n} h(n)$, where $h$ is a subexponential factor, i.e., $\lim _{\sup _{n \rightarrow \infty}}|h(n)|^{1 / n}=1$.

Proof: (of Theorem 2.4) We use the theory of singularity analysis from Flajolet and Sedgewick (2009). In particular, we use Theorem VI.5 from page 398 of Flajolet and Sedgewick (2009), which we quote:

Theorem VI.5 (Singularity analysis, multiple singularities). Flajolet and Sedgewick (2009)

Let $f(z)$ be analytic in $|z|<\rho$ and have a finite number of singularities on the circle $|z|=\rho$ at points $\zeta_{j}=\rho e^{\mathrm{i} \theta_{j}}$, for $j=1 . . r$. Assume that there exists a $\Delta$-domain $\Delta_{0}$ such that $f(z)$ is analytic in the indented disc

$$
\mathbf{D}=\bigcap_{j=1}^{r}\left(\zeta_{j} \cdot \Delta_{0}\right)
$$

with $\zeta \cdot \Delta_{0}$ the image of $\Delta_{0}$ by the mapping $z \mapsto \zeta z$.

Assume that there exists $r$ functions $\sigma_{1}, \ldots, \sigma_{r}$, each a linear combination of elements from the scale $\mathcal{S}$, and a function $\tau \in \mathcal{S}$ such that

$$
f(z)=\sigma_{j}\left(z / \zeta_{j}\right)+O\left(\tau\left(z / \zeta_{j}\right)\right) \quad \text { as } z \rightarrow \zeta_{j} \text { in } \mathbf{D} .
$$

Then the coefficients of $f(z)$ satisfy the asymptotic estimate

$$
f_{n}=\sum_{j=1}^{r} \zeta_{j}^{-n} \sigma_{j, n}+O\left(\rho^{-n} \tau_{n}^{\star}\right)
$$

where each $\sigma_{j, n}=\left[z^{n}\right] \sigma_{j}(z)$ has its coefficients determined by Theorems VI.1, VI.2 and $\tau_{n}^{\star}=$ $n^{a-1}(\log n)^{b}$, if $\tau(z)=(1-z)^{-a} \lambda(z)^{b}$.

For the function $f(z)=\frac{\arctan \sqrt{2 e^{-z}-1}}{\sqrt{2 e^{-z}-1}}$ in Wilf's problem, $f(z)$ is analytic in the open disc $\mathcal{C}_{R}:=$ $\{z|| z \mid<R\}$. On the boundary of $\mathcal{C}_{R}$, the function $f(z)$ has non-removable singularities at $z=\zeta, \bar{\zeta}$. In 
the notation in Flajolet and Sedgewick (2009), we have $\zeta_{1}=\zeta$ and $\zeta_{2}=\bar{\zeta}$, as well as $\rho=R$. We define the $\Delta$-domain

$$
\Delta_{0}=\left\{z|| z\left|<\frac{|\ln (2)+6 \pi \mathrm{i}|}{|\ln (2)+2 \pi \mathrm{i}|}, \quad z \neq 1, \quad\right| \arg (z-1) \mid>\arctan (2 \pi / \ln (2))\right\} .
$$

By using the domain in which $f(z)$ is analytic, established in Lemma 2.2 we see that $f(z)$ is analytic throughout the domain $\mathbf{D}=\left(\zeta \cdot \Delta_{0}\right) \cap\left(\bar{\zeta} \cdot \Delta_{0}\right)$. Fix a positive integer $N$. Then we can define

$$
\begin{aligned}
\sigma_{m}\left(z / \zeta_{m}\right) & =-\frac{\pi}{\sqrt{\sum_{j=1}^{N}\left(\zeta_{m}-z\right)^{j} / j !}} \quad \text { for } m=1,2 \text { (i.e., for } \zeta_{1}=\zeta \text { and } \zeta_{2}=\bar{\zeta} \text { ), } \\
\tau(z) & =(1-z)^{N-1 / 2},
\end{aligned}
$$

and we have the required conditions

$$
f(z)=\sigma_{m}\left(z / \zeta_{m}\right)+O\left(\tau\left(z / \zeta_{m}\right)\right) \quad \text { as } z \rightarrow \zeta_{m} \text { in } \mathbf{D}, \text { for } m=1,2 .
$$

Let $d_{j}=\left[z^{j-1 / 2}\right]\left(e^{z}-1\right)^{-1 / 2}$, so $\left\{d_{0}, d_{1}, d_{2}, \ldots\right\}=\left\{1,-\frac{1}{4}, \frac{1}{96}, \ldots\right\}$. (Note that $\left(e^{z}-1\right)^{-1 / 2}$ is the square root of an exponential generation function for the Bernoulli numbers.) Then

$$
\sigma_{m}\left(z / \zeta_{m}\right)=-\pi \sum_{j=0}^{N-1} d_{j}\left(\zeta_{m}-z\right)^{j-1 / 2}+O\left(\zeta_{m}-z\right)^{N-1 / 2}, \quad \text { for } m=1,2 .
$$

For instance, if $N=3$,

$\sigma_{m}\left(z / \zeta_{m}\right)=-\pi\left(\left(\zeta_{m}-z\right)^{-1 / 2}-\frac{1}{4}\left(\zeta_{m}-z\right)^{1 / 2}+\frac{1}{96}\left(\zeta_{m}-z\right)^{3 / 2}\right)+O\left(\zeta_{m}-z\right)^{5 / 2}, \quad$ for $m=1,2$.

The explicit asymptotic expansions $(1-z)^{-\alpha}$ are also well-known (we follow the notation from pages 381-384 of Flajolet and Sedgewick (2009)),

$$
\left[z^{n}\right](1-z)^{-\alpha} \sim \frac{n^{\alpha-1}}{\Gamma(\alpha)}\left(1+\sum_{k=1}^{\infty} \frac{e_{k}(\alpha)}{n^{k}}\right)
$$

where $e_{k}(\alpha)$ is a sum of polynomials in $\alpha$ of degrees between $k$ and $2 k$ (thus, the overall degree of $e_{k}(\alpha)$ is $2 k$ ),

$$
e_{k}(\alpha)=\sum_{\ell=k}^{2 k}(-1)^{\ell} \lambda_{k, \ell}(\alpha-1)(\alpha-2) \cdots(\alpha-\ell),
$$

and $\lambda_{k, \ell}=\left[v^{k} t^{\ell}\right] e^{t}(1+v t)^{-1-1 / v}$. Thus, the coefficients $a_{n}$ of $f(z)$ satisfy the asymptotic estimate

$$
a_{n}=-\pi \sum_{j=0}^{N-1} d_{j} \frac{\zeta^{-n+j-1 / 2}+(\bar{\zeta})^{-n+j-1 / 2}}{\Gamma(-j+1 / 2) n^{j+1 / 2}}\left(1+\sum_{k=1}^{N-j-1} \frac{e_{k}}{n^{k}}\right)+O\left(\frac{1}{R^{n} n^{N+1 / 2}}\right)
$$


In the case $N=3$, we explicitly compute the asymptotic expansions:

$$
\begin{aligned}
{\left[z^{n}\right](1-z)^{-1 / 2} } & =\frac{1}{\sqrt{\pi n}}\left(1-\frac{1}{8 n}+\frac{1}{128 n^{2}}+O\left(n^{-3}\right)\right) \\
{\left[z^{n}\right](1-z)^{1 / 2} } & =-\frac{1}{\sqrt{\pi n^{3}}}\left(\frac{1}{2}+\frac{3}{16 n}+O\left(n^{-2}\right)\right) \\
{\left[z^{n}\right](1-z)^{3 / 2} } & =\frac{1}{\sqrt{\pi n^{5}}}\left(\frac{3}{4}+O\left(n^{-1}\right)\right) .
\end{aligned}
$$

It follows that the coefficients $a_{n}$ of $f(z)$ satisfy the asymptotic estimate

$$
\begin{aligned}
a_{n}=-\sqrt{\pi} & {\left[\left(\frac{1}{\zeta^{n+1 / 2}}+\frac{1}{(\bar{\zeta})^{n+1 / 2}}\right)\left(\frac{1}{\sqrt{n}}-\frac{1}{8 n^{3 / 2}}+\frac{1}{128 n^{5 / 2}}\right)\right.} \\
& \left.+\left(\frac{1}{\zeta^{n-1 / 2}}+\frac{1}{(\bar{\zeta})^{n-1 / 2}}\right)\left(\frac{1}{8 n^{3 / 2}}+\frac{3}{64 n^{5 / 2}}\right)+\left(\frac{1}{\zeta^{n-3 / 2}}+\frac{1}{(\bar{\zeta})^{n-3 / 2}}\right)\left(\frac{3}{384 n^{5 / 2}}\right)\right] \\
& +O\left(\frac{1}{R^{n} n^{7 / 2}}\right) .
\end{aligned}
$$

To simplify these expressions, we note that, for $a=n-j-1 / 2$,

$$
\begin{aligned}
R^{a}\left(\zeta^{-a}+(\bar{\zeta})^{-a}\right) & =(\bar{\zeta} / \zeta)^{a / 2}+(\zeta / \bar{\zeta})^{a / 2} \\
& =\exp \left(\frac{a}{2} \log (\bar{\zeta} / \zeta)\right)+\exp \left(-\frac{a}{2} \log (\bar{\zeta} / \zeta)\right) \\
& =\exp \left(-\mathrm{i} a \frac{\mathrm{i}}{2} \log \frac{\mathrm{i}+\frac{2 \pi}{\ln (2)}}{\mathrm{i}-\frac{2 \pi}{\ln (2)}}\right)+\exp \left(\mathrm{i} a \frac{\mathrm{i}}{2} \log \frac{\mathrm{i}+\frac{2 \pi}{\ln (2)}}{\mathrm{i}-\frac{2 \pi}{\ln (2)}}\right) \\
& =\exp (-\mathrm{i} a \arctan (2 \pi / \ln (2)))+\exp (\mathrm{i} a \arctan (2 \pi / \ln (2))) \\
& =2 \cos (a \arctan (2 \pi / \ln (2)))
\end{aligned}
$$

So it follows that

$a_{n}=-2 \pi \sum_{j=0}^{N-1} d_{j} \frac{\cos ((n-j+1 / 2) \arctan (2 \pi / \ln (2)))}{\Gamma(-j+1 / 2) R^{n-j+1 / 2} n^{j+1 / 2}}\left(1+\sum_{k=1}^{N-j-1} \frac{e_{k}\left(\frac{1}{2}-j\right)}{n^{k}}\right)+O\left(\frac{1}{R^{n} n^{N+1 / 2}}\right)$.

For example, when $N=3$,

$$
\begin{aligned}
a_{n}=-2 \sqrt{\pi} & {\left[\frac{\cos ((n+1 / 2) \arctan (2 \pi / \ln (2)))}{R^{n+1 / 2}}\left(\frac{1}{\sqrt{n}}-\frac{1}{8 n^{3 / 2}}+\frac{1}{128 n^{5 / 2}}\right)\right.} \\
& +\frac{\cos ((n-1 / 2) \arctan (2 \pi / \ln (2)))}{R^{n-1 / 2}}\left(\frac{1}{8 n^{3 / 2}}+\frac{3}{64 n^{5 / 2}}\right) \\
& \left.+\frac{\cos ((n-3 / 2) \arctan (2 \pi / \ln (2)))}{R^{n-3 / 2}}\left(\frac{3}{384 n^{5 / 2}}\right)\right] \\
& +O\left(\frac{1}{R^{n} n^{7 / 2}}\right) .
\end{aligned}
$$

For more terms in the asymptotic expansion, the method of analysis proceeds in exactly the same way. 


\section{Acknowledgements}

The author sincerely thanks Herb Wilf for posting this very interesting problem on his webpage, for a short conversation about the problem at AofA 2009, and for insightful discussions during the author's visit to the University of Pennsylvania after submitting the solution. The author is very thankful for discussions prior to submission with a few colleagues about this problem, namely, Steve Bell, Michael Drmota, Philippe Flajolet, Bruno Salvy, and Wojciech Szpankowski. The author also warmly thanks Pierre Nicodème and Mireille Régnier, who graciously hosted the author during his recent visit to Paris in November 2009. Between the initial submission and the final version for publication, the author was fortunate to get several insightful questions and suggestions from Alex Eremenko, Andrei Gabrielov, and Robin Pemantle. The author also appreciates constructive, helpful advice garnered from three anonymous reviewers. The author is thankful to all of these people for the resulting improvements to the content and presentation of the paper.

\section{References}

L. V. Ahlfors. Complex Analysis. McGraw-Hill, New York, 3rd edition, 1979.

J. W. Brown and R. V. Churchill. Complex Variables and Applications. McGraw-Hill, 8th edition, 2009.

P. Flajolet and R. Sedgewick. Analytic Combinatorics. Cambridge, 2009.

J. Hadamard. The Psychology of Invention in the Mathematical Field. Princeton, 1945.

P. Henrici. Applied and Computational Complex Analysis (3 volumes). Wiley, New York, 1974, 1977, 1986.

P. Painlevé. Analyse des travaux scientifiques jusqu'en 1900. Gauthier-Villars, 1900.

W. Szpankowski. Average Case Analysis of Algorithms on Sequences. Wiley, New York, 2001.

H. Wilf. Some unsolved problems, September 2009. Available for download as an electronic pdf file, http://www.math.upenn.edu/ wilf/website/Unsolvedproblems.pdf. 
